\title{
Applicability and Effectiveness of Ultrasound Combined Nerve Stimulator-Guided Lumbosacral Plexus Block in Supine Versus Lateral Position During Surgeries for Lower Limb Fracture-A Prospective Randomized Controlled Trial
}

\section{Yuting Xu}

Chaohu Hospital Affiliated to Anhui Medical University

Jie Song the Second Hospital Affiliated to Anhui Medical University

Xiaoqiong Xia ( $\nabla x x$ 2366833@sina.com )

Chaohu Hospital Affiliated to Anhui Medical University

Xianwen Hu

the Second Hospital Affiliated to Anhui Medical University

Yawen Li

Chaohu Hospital Affiliated to Anhui Medical University

Yongbo Yu

Chaohu Hospital Affiliated to Anhui Medical University

Liang Wang

Chaohu Hospital Affiliated to Anhui Medical University

\section{Zhiguo Tao}

Chaohu Hospital Affiliated to Anhui Medical University

\section{Research Article}

Keywords: Nerve block, Nerve stimulation, Ultrasound, lumbosacral plexus block, Lower Extremity

Posted Date: February 7th, 2022

DOI: https://doi.org/10.21203/rs.3.rs-1157712/v1

License: (c) (i) This work is licensed under a Creative Commons Attribution 4.0 International License. Read Full License 


\section{Abstract}

Background:To compare the applicability and effectiveness of ultrasound combined nerve stimulatorguided lumbosacral plexus block (LSPB) in supine versus lateral position during the lower limb fracture surgery.

Methods:Patients who underwent elective internal fixation for lower limb fracture were divided into the S group and the L group by the random number table method and received LSPB guided by ultrasound combined nerve stimulator in supine and lateral positions, respectively. The primary outcome examined was the dose of sufentanil used in surgery and the secondary outcomes were the maximum VAS pain score at position placing for LSPB, the time of position placing, the time for nerve block and the number of puncture attempts.

Results:The maximum pain score at position placing, the time of position placing for nerve block, the time for lumbar plexus block and the time of puncture attempts were significantly lower in the $\mathrm{S}$ group compared with those of the $\mathrm{L}$ group (all $P<0.05$ ). However, the time for sacral plexus block was higher in the $S$ group compared with that of the $L$ group $(P<0.05)$. The heart rate and mean arterial pressure at different time points, times of puncture attempts for sacral plexus, dose of sufentanil used, VAS scores, the satisfactory postoperative degree to analgesia and adverse events of nerve block did not significantly differ between the two groups (all $P>0.05$ ).

Conclusions:LSPB in the supine position is simple to apply and has definite anesthetic effects. Meanwhile, it has a high comfort level and therefore should be widely applied. The trial was registered prior to patient enrollment at the Chinese Clinical Trail Registry (Date:11/03/2021 Number: ChiCTR2100044117).

\section{Background}

Lower limb fractures account for approximately one third of all fractures and may result in substantial mortality and morbidity [1] The most common anatomic position of lower limb fracture is the ankle joint, which accounts for $22.6 \%$ of all lower limb fractures, followed by tibia/fibula (17.3\%), hip joint (16.7\%) and tarsal/metatarsal bone (16.7\%). Fractures of the hip, femur and other parts account for approximately $25 \%$ of all fractures [2]. A previous study by Kaye and Jick demonstrated [2] the largest relative risk estimate observed for road collisions. In addition, certain studies have shown that a number of risk factors have been identified for fractures including previous fragility fracture [3], smoking and alcohol consumption and falls and medication use. Certain medications have been proposed as risk factors for fractures, including oral glucocorticoids [4], bisphosphonates [5] and psychotropic drugs (opiates, benzodiazepines, anticholinergic agents, dopaminergic agents and antidepressants) [6].

Rapid urbanization and accelerated aging of the population in China have led to a rapid increase in the number of patients with fractures caused by traffic injuries, architecture injuries and senile osteoporosis. The increase in residual injuries and disability increases the potential life loss and thus has become an 
important public health concern [7]. Lower limb fractures include various types and are usually caused by large wounds. Therefore they generally require correction by surgery.

Over the recent years, lumbosacral plexus block (LSPB) has been widely applied in orthopedics departments due to the advantages, including reduction in the application of opiates, decreasing the occurrence of acute pain, promoting early activation and shortening the time of hospital stay [8]. De Visme et al. has proved that LSPB exhibit an advantage of limited sympathectomy and bladder paralysis [9]. Ultrasound-guided nerve blocks with nerve stimulators increase the success rate and reduce risks, such as nerve injuries, undesirable spread-hematoma and renal puncture [10] The conventional LSPB is mainly performed in the lateral position, while only very few studies have reported LSPB in the supine position. Therefore, the present study aimed to compare the applicability, anesthetic effects and postoperative analgesic effects of supine versus lateral LSPB under the guidance of ultrasound combined nerve stimulator and provide evidence for the application of LSPB in a supine position for anesthesia in clinical practice.

\section{Methods}

\section{General characteristics}

This randomized, blinded study was performed in compliance with the declaration of Helsinki and its amendments and was conducted according to the principles of Good Clinical Practice. The trial was registered prior to patient enrollment at the Chinese Clinical Trail Registry (11/03/2021

ChiCTR2100044117). The present study was approved by the Ethics Committee of the Chaohu Hospital Affiliated to Anhui Medical University (01/12/2020 202001-KYXM-01) and all patients signed the relevant informed consent form. A total of 126 patients with the following fractures were recruited at the department of Anesthesiology in two affiliated hospitals of Anhui Medical University (Chaohu Hospital, Chaohu; and the Second Hospital, Hefei). The patients were individually randomized by the hospital. The patients who underwent elective internal fixation for unilateral lower limb fracture (ascertained using radiological reports from the medical imaging centre, at Chaohu Hospital and the Second Hospital) between March 2021 and June 2021, were included.

\section{Inclusion and exclusion criteria}

The inclusion criteria used were the following: 1) Patients diagnosed with unilateral femoral neck fracture or lower fractures by X-ray or CT examination; 2) patients who consented to participate in the study and signed the relevant informed consent form (a study physician approached the patient in the preoperative area and informed consent was obtained preoperatively if he or she elected to proceed with the study); 3) patients with available complete clinical data and 4) patients with the capability of communication, expression and comprehension. 
The exclusion criteria were the following: 1) Patients with mental disorders or psychonosema; 2) patients accompanied with renal, liver or cardiac insufficiency; 3 ) patients with coagulation disorders; 4) patients who refused to participate or withdrew due to personal reasons.

\section{Treatment methods}

\section{Methods of anesthesia}

All the patients received vital sign monitoring following entry in the operating room and the venous channel was established. Prior to establishing the venous channel, intravenous pumping of Dexmedetomidine (Yangzijiang Pharmaceutical Group Co. Ltd; SFDA approval number: H20183220) was performed at the rate of $300 \mu \mathrm{g} / \mathrm{h}$ for $10 \mathrm{~min}$ to induce the full sedation of the patients. Subsequently, LSPB was performed under the guidance of ultrasound combined nerve stimulator, using nerve stimulator (Stimuplex HNS 12, B. BRAUN, Germany) with 5-10 MHz high-frequency linear array probe and 2-5 MHz low-frequency convex array probe (FUJIFILM SonoSite, Bothell, WA98021 USA). Ropivacaine was from Xianju Pharmaceutical Group Co. Ltd (Zhejiang; SFDA approval number: H20163208).

This trial was a randomized controlled single blinded clinical trial. The patients were blind to the group allocations. Group assignments were concealed in opaque envelopes until consent had been obtained.

\section{Treatment of the S group}

The anterior approach block of the lumbar plexus and fascia iliaca compartment block above inguen were performed for patients of the S group. In brief [11], the superior anterior spine was touched and the high-frequency linear array probe was placed on the sagittal plane to acquire images of the anterior superior spine (Fig. 2A). The probe was slid inward to detect the iliac muscle. Subsequently, the probe was adjusted for ultrasound anatomy, including the detection of subcutaneous tissues, obliquus internus abdominis, sartorius muscle, iliac fascia, and iliac muscle (Fig. 2B). Intra-plane insertion of the needle was performed by inserting a 21-G $100 \mathrm{~mm}$ needle to puncture the iliac fascia. The needlepoint was allowed to reach the site below the iliac fascia and no gas or blood was found in back-pumping. A total of $5 \mathrm{ml}$ normal saline was injected to clarify the site of the needle point. After the site was considered appropriate, the water separation technique was adopted by gradual injection of $30 \mathrm{ml} 0.3 \%$ ropivacaine into the superficial and deep sites of the iliac muscle to expand the iliac fascia. For sacral plexus block in the supine position [12], the patients adopted the supine position with the affected side uplifted by $15^{\circ}$. A line from the anterior superior spine was made vertical to the midaxillary line and the intersection point was considered the positioning point (Fig. 2C). The maximal axis of the low-frequency convex array probe was vertical to the midaxillary line, after which the probe was slid from the head end to the tail end and was terminated when the iliac bone continuously appeared. The sacral plexus nerve was in the hyperecho area posterior to the iliac bone (Fig. 2D). Extra-plane needle insertion was performed. Under the guidance of ultrasound, the needlepoint was inserted toward the sacral plexus, whereas the initial current of the nerve stimulator, which was set at $1 \mathrm{~mA}$, could induce the movement of tensor fasciae latae when reaching the sacral plexus. Subsequently, the current was adjusted to $0.5 \mathrm{~mA}$ and the needle was slowly 
inserted. When contraction of calf muscles was induced and back-pumping indicated lack of gas or blood, $15-20 \mathrm{ml} 0.3 \%$ ropivacaine was injected.

\section{Treatment of the L group}

The lumbar plexus block for patients in the $L$ group was performed according to previous studies [13]. The patients were placed in the lateral position and the skin and ultrasound probe were disinfected. The maximal axis of the low-frequency convex array probe was placed at the L3-4 space and at $4 \mathrm{~cm}$ parallel to the posterior midline of the spine (Fig. 2E). The ultrasound image indicated a trident-shaped structure formed by the transverse processes of three lumbar vertebrae. The transverse processes of the L3-4 were positioned and the ultrasound probe was moved until the midpoint of the line between L3 and L4 transverse processes was located on the midline of the screen of the ultrasound apparatus (Fig. 2F). Extra-plane needle insertion was performed at the midpoint of the ultrasound probe until the needle point reached $1.5 \mathrm{~cm}$ below the transverse process and until it was superior to the musculiinter transversarii laterals. The nerve stimulator was connected to the nerve for nerve stimulation. Following contraction of quadriceps femoris muscle, the current was adjusted to $0.5 \mathrm{~mA}$; in case of absence of blood or cerebrospinal fluid (indicated by back-pumping) and lack of contraction of the quadriceps femoris muscle, $25-30 \mathrm{ml}$ ropivacaine $(0.3 \%)$ was injected and intermittent back-pumping was performed to avoid intravenous injection. The midpoint of the line from the upper margin of the greater trochanter of femur was marked to the posterior superior iliac spine in order to induce the sacral plexus block in a lateral position[14], from which a vertical line was made (an inner-downward line). This line met the line from the greater trochanter of femur to sacral hiatus and the intersection was the positioning point for the sacral plexus (Fig. 2E). The probe was routinely disinfected and subsequently placed at this intersection with the low-frequency convex array probe parallel to the line between the greater trochanter of the femur to the sacral hiatus. The image of the slope-shaped sacroiliac joint was displayed by ultrasound and the probe was slid along the line toward the tail end until the sacroiliac joint disappeared. Subsequently, the sonographic images of the ischium in the outer side and of the sacrum in the inner side were displayed and the hyper-echo between the ischium and sacrum was the sacral plexus (Fig. 2G). The sacral plexus block was performed under the assistance of a nerve stimulator as conducted in the patients of the $S$ group.

All the nerve blocks were performed by the same experienced attending anesthetist. Following completion of the nerve block in both groups, venous anesthesia was performed. In brief, $0.2 \mu \mathrm{g} / \mathrm{kg}$ sufentanil and $1.5-2 \mathrm{mg} / \mathrm{kg}$ propofol were used for anesthesia induction. Intubation was performed via laryngeal mask based on a BIS value of $<60$ to allow autonomous or controlled respiration. For anesthesia maintenance, venous pumping of propofol [4-12 mg/(kg.h)] was performed. According to the hemodynamics, $5 \mu \mathrm{g}$ sufentanil was added if the heart rate or arterial pressure was increased by $15 \%$ in the surgery. Following surgery, an analgesia pump (8-10 mg butorphanol +10 mgazasetron, diluted to $100 \mathrm{ml}$ ) was used for analgesia, with the initial dose of $2 \mathrm{ml}$, background dose of $2 \mathrm{ml} / \mathrm{l}$, PCA dose of $2 \mathrm{ml}$ and limiting dose of $18 \mathrm{ml} / \mathrm{h}$. 
The operation was performed by four medical groups in the two hospitals. The chief surgeon was a deputy chief physician who was engaged in orthopedic surgery for more than 10 years.

\section{Evaluation indicators}

An investigator recorded (1) the maximum VAS pain score at position placing for LSPB, time of position placing, time for nerve block and a number of puncture attempts during the nerve block; (2) subsequently the dose of sufentanil used and hemodynamic indicators during the surgery were recorded; (3) the VAS score at 1, 12, and $24 \mathrm{~h}$ following surgery, postoperative satisfactory degree to analgesia, nerve block results and adverse events were also recorded in the two groups.

The following evaluation indicators were used: The primary outcome was (1) the dose of sufentanil used in surgery. The secondary outcomes were (2) the maximum VAS pain score at position placing for LSPB (1-10 points: 0 point indicated no pain and 10 points indicated drastic pain); the time of position placing, the time for nerve block (from skin anesthesia, ultrasound imaging, to the completion of local anesthetics injection) and a number of puncture attempts (each withdrawal of the needle to adjust the direction was considered as one attempt of puncture); (3) the hemodynamic indicators, including heart rate and arterial pressure and the observation time including the time of entering the operating room (T0), completion of a nerve block (T1), skin incision (T2), skin suturing (T3) and 30 min following completion of the surgery (T4); (4) the postoperative VAS score, with the higher scores indicating more severe pain. The VAS score was evaluated at 1, 12, and $24 \mathrm{~h}$ following surgery; (5) the postoperative satisfactory degree to analgesia, where the scores ranged from 1 to 4 points (1, poor; 2, fair; 3, satisfactory; and 4, highly satisfactory); (6) the number of patients with postoperative nausea and vomiting, the toxicity of local anesthetics, hematoma at the puncture site and incidence of postoperative epidural volume extension.

\section{Statistical analysis}

IBM SPSS Statistics 24.0(Version24; IBM, Armonk, New York) software was used for statistical analysis. Continuous variables were expressed as mean and variance and analyzed using an independent samples $t$-test if the data were normally distributed. In case of non-normal distribution, the results were expressed as median and range and analyzed with the Mann-Whitney $U$ test. The categorical variables were expressed as percentages or numbers and analyzed by the Pearson's Chi-square tests or the Fisher's exact test. The significance level for all statistical tests was set at $P<0.05$.

The present study was a randomized control trial with a non-inferiority design, which aimed to investigate whether the effectiveness of the anterior approach LSPB was not inferior to the conventional LSPB. According to the previous trial findings, the dose of sufentanil was approximately $26.4 \pm 4.2 \mu \mathrm{g}$ in the conventional LSPB. For the anterior approach LSPB, the standard sufentanil dose was $3.7 \mu \mathrm{g}$ for the anterior approach LSPB. The cut-off value of inferiority ( $\delta$ ) was $2.1 \mu \mathrm{g}$, whereas the a value was 0.025 (one-sided) and the power (1- $\beta$ ) was 0.8 . The numbers of subjects in the two groups were equal. PASS 11 software was used to estimate the sample size in the study and control groups, generating N1=N2=57. A drop-off ratio of $10 \%$ was considered and the final sample size was 126 (63 per group). 


\section{Results}

A total of 140 patients were screened (Fig. 1), of whom 14 were excluded. A total of 7 out of these 14 patients did not meet the inclusion criteria, 4 declined to participate and 3 were unable to consent. The study cohort included 126 patients. A computer-generated allocation program randomly assigned the patients to the $S$ and $L$ groups, with 63 patients receiving the LSPB in the supine and lateral positions, respectively.

\section{Comparison of general characteristics of patients between the two groups}

A total of 126 patients with the following fractures were included: femoral neck fracture in 26 patients, femoral intertrochanteric fracture in 18 patients, femoral shaft fracture in 20 patients, tibial plateau fracture in 17 patients, tibial and fibula shaft fracture in 30 patients, malleolus fracture in 15 patients. The age, BMI, ASA grade and sex were not significantly different between the two groups $(P>0.05)$ (Insert Table 1 here).

Table 1

Comparison of general characteristics of the patients between the two groups (mean \pm standard)

\begin{tabular}{|lllll|}
\hline Parameter & S group $(\mathbf{n = 6 3})$ & L group $(\mathbf{n = 6 3})$ & Statistics & P value \\
\hline Age (years) & $56.6 \pm 15.4$ & $55.8 \pm 14.6$ & $\mathrm{t}=0.309$ & 0.758 \\
\hline BMI (Kg/ $\mathbb{\text { }})$ & $22.8 \pm 1.8$ & $23.0 \pm 2.0$ & $\mathrm{t}=-0.579$ & 0.564 \\
\hline ASA grade I/II (n) & $27 / 36$ & $25 / 38$ & $\chi 2=0.131$ & 0.717 \\
\hline Sex (M/F) & $38 / 25$ & $34 / 29$ & $\chi 2=0.519$ & 0.471 \\
\hline Note: BMI ,Body Mass Index; ASA, American Society of Anesthesiologists \\
\hline
\end{tabular}

\section{Comparison of the parameters of LSPB and intraoperative sufentanil dose between the two groups}

The maximum pain score at position placing, time of position placing, time for Lumbar plexus block and the number of puncture attempts for Lumbar plexus were significantly lower in the $S$ group compared with those of the $L$ group $\left(P_{1}<0.01, P_{2}<0.01, P_{3}<0.01, P_{5}<0.01\right)$. However, the time for sacral plexus block was significantly longer in the $S$ group than that noted in the $L$ group $\left(P_{4}=0.029\right)$. The number of puncture attempts for sacral plexus and dose of sufentanil used in surgery did not significantly differ between the two groups $\left(P_{6}=0.802, P_{7}=0.142\right)$ (Insert Table 2 here). 
Table 2

Comparison of parameters of LSPB and intraoperative sufentanil dose between the two groups (mean \pm standard, median (25-75 IQ))

\begin{tabular}{|c|c|c|c|c|c|}
\hline \multicolumn{2}{|l|}{ Parameter } & $\begin{array}{l}\text { S group } \\
(n=63)\end{array}$ & $\begin{array}{l}\text { L group } \\
(n=63)\end{array}$ & Statistics & $P$ value \\
\hline \multicolumn{2}{|l|}{ VAS score at position placing } & $2.0(1.0,2.0)$ & $4 \cdot 0(3 \cdot 0,4 \cdot 0)$ & $Z=-9.070$ & $P 1<0.01$ \\
\hline \multicolumn{2}{|l|}{ Time of position placing (S) } & $48.8 \pm 8.9$ & $113.8 \pm 8.0$ & $t=-43.161$ & $P 2<0.01$ \\
\hline \multirow[t]{2}{*}{ Time for nerve block (S) } & $\begin{array}{l}\text { Lumbar } \\
\text { plexus }\end{array}$ & $236.9 \pm 12.0$ & $318.7 \pm 13.1$ & $t=-36.478$ & $P 3<0.01$ \\
\hline & $\begin{array}{l}\text { Sacral } \\
\text { plexus }\end{array}$ & $304.4 \pm 11.1$ & $300.3 \pm 9.9$ & $t=2.207$ & $P 4=0.029$ \\
\hline \multirow[t]{2}{*}{$\begin{array}{l}\text { Time of puncture attempts } \\
\text { (time) }\end{array}$} & $\begin{array}{l}\text { Lumbar } \\
\text { plexus }\end{array}$ & $1.0(1.0,1.0)$ & $2 \cdot 0(1 \cdot 0,3.0)$ & $Z=-6.031$ & $P 5<0.01$ \\
\hline & $\begin{array}{l}\text { Sacral } \\
\text { plexus }\end{array}$ & $2.0(2.0,3.0)$ & $2.0(2.0,3.0)$ & $Z=-0.251$ & $P 6=0.802$ \\
\hline \multicolumn{2}{|l|}{ Dose of sufentanil $(\mu \mathrm{g})$} & $25.1 \pm 3.1$ & $26.0 \pm 3.0$ & $t=-1.479$ & $P 7=0.142$ \\
\hline
\end{tabular}

\section{Comparison of hemodynamic indicators at different time points between the two groups}

The heart rate and MAP at the time points of T0, T1, T2, T3 and T4 did not significantly differ between the $S$ group and $L$ groups $\left(P_{1}=0.413, P_{2}=0.656, P_{3}=0.117, P_{4}=0.880, P_{5}=0.642, P_{6}=0.146, P_{7}=0.446, P_{8}=0.688\right.$, $\left.P_{9}=0.430, P_{10}=0.237\right)($ Insert Table 3 here). 
Table 3

Comparison of hemodynamic indicators at different time points between the two groups (mean \pm standard)

\begin{tabular}{|llllll|}
\hline Parameter & & S group $(n=63)$ & L group $(n=63)$ & $t$ & $P$ value \\
\hline Heart rate (beats/min) & T0 & $75.6 \pm 7.5$ & $76.7 \pm 7.0$ & $t=-0.821$ & $P 1=0.413$ \\
& T1 & $63.6 \pm 4.8$ & $64.0 \pm 4.7$ & $t=-0.446$ & $P 2=0.656$ \\
& T2 & $64.3 \pm 4.0$ & $65.7 \pm 5.7$ & $t=-1.581$ & $P 3=0.117$ \\
& T3 & $68.2 \pm 5.0$ & $68.3 \pm 5.5$ & $t=-0.152$ & $P 4=0.880$ \\
& T4 & $77.7 \pm 6.2$ & $78.2 \pm 6.8$ & $t=-0.466$ & $P 5=0.642$ \\
Mean arterial pressure $(\mathrm{mmHg})$ & T0 & $94.6 \pm 9.7$ & $92.1 \pm 9.3$ & $t=1.462$ & $P 6=0.146$ \\
& T1 & $86.7 \pm 9.8$ & $85.3 \pm 10.1$ & $t=0.765$ & $P 7=0.446$ \\
& T2 & $85.1 \pm 7.8$ & $85.7 \pm 8.2$ & $t=-0.402$ & $P 8=0.688$ \\
& T3 & $90.3 \pm 9.2$ & $89.0 \pm 8.7$ & $t=0.792$ & $P 9=0.430$ \\
& T4 & $95.1 \pm 9.9$ & $93.0 \pm 9.1$ & $t=1.188$ & $P 10=0.237$ \\
\hline
\end{tabular}

Comparison of postoperative VAS score and satisfactory degree to analgesia at different time points between the two groups

The VAS score and satisfactory degree to analgesia at different time points following operation were not significantly different between the two groups $\left(P_{1}=0.609, P_{2}=0.361, P_{3}=0.189, P_{4}=0.683\right)$ (Insert Table 4 here). 
Table 4

Comparison of postoperative VAS score and satisfactory degree to analgesia at different time points between the two groups (median (25-75 IQ))

\begin{tabular}{|llllll|}
\hline Parameter & $\begin{array}{l}\text { S group } \\
(\mathbf{n = 6 3 )}\end{array}$ & $\begin{array}{l}\text { L group } \\
(\mathbf{n}=63)\end{array}$ & $Z$ & $P$ value \\
\hline $\begin{array}{l}\text { Postoperative VAS } \\
\text { score }\end{array}$ & $\begin{array}{l}\text { 1 h after } \\
\text { operation }\end{array}$ & $1.0(1.0,2.0)$ & $1.0(1.0,2.0)$ & $Z=-0.511$ & $P 1=0.609$ \\
\cline { 2 - 5 } & $\begin{array}{l}\text { 12 h after } \\
\text { operation }\end{array}$ & $3.0(3.0,4.0)$ & $3.0(3.0,4.0)$ & $\begin{array}{l}Z \\
=-0.914\end{array}$ & $P 2=0.361$ \\
\hline $\begin{array}{l}\text { 24 h after } \\
\text { operation }\end{array}$ & $5.0(5.0,6.0)$ & $5.0(5.0,6.0)$ & $\begin{array}{l}Z \\
=-1.313\end{array}$ & $P 3=0.189$ \\
\hline $\begin{array}{l}\text { Postoperative satisfactory degree to } \\
\text { analgesia }\end{array}$ & $4.0(3.0,4.0)$ & $4.0(3.0,4.0)$ & $\begin{array}{l}Z \\
=-0.409\end{array}$ & $P 4=0.683$ \\
\hline Note: VAS visual analogue scale & & & & \\
\hline
\end{tabular}

\section{Comparison of nerve block results and adverse events between the two groups}

No adverse events, including the toxicity of local anesthetics, hematoma at puncture sites and postoperative epidural volume extension, were found. Eight patients $(12.7 \%)$ in the S group and $6(9.5 \%)$ patients in the $L$ group suffered from nausea and vomiting; however, the difference between the two groups was not statistically significant (all $P>0.05$ ).

\section{Discussion}

Two main findings can be derived from our prospective randomized controlled trial. Firstly, the data indicated that LSPB in the supine position could reduce the maximum pain score at position placing and time of position placing and ensure a high comfort level. Secondly, supra-inguinal fascia iliaca (SIFI) block shortened the time periods required for nerve block and puncture and reduced the possibility of tissue injury and nerve damage compared with those noted in the traditional lumbar nerve block.

Anesthesia methods for internal fixation in patients with lower limb fracture included simple general anesthesia, intraspinal anesthesia and general anesthesia combined with a nerve block. Modern anesthesia should not only ensure successful surgical processes but also take into account the comfort of patients. With the development of visualization technology and the application of nerve stimulators, nerve block [15] has become increasingly and widely used in clinical practice due to specific advantages, including high safety, low invasiveness and low side effects. General anesthesia combined with nerve block has been widely used in orthopedic surgeries due to its well-recognized advantages.

Conventional LSPB is performed in the lateral position, where patients are required to engage in position changing and is therefore not convenient for patients treated with an external fixator. The classic anterior 
approach for blocking the lumbar plexus [16] could easily lead to abdominal visceral injuries due to the deep position, which could in turn induce complications, such as epidural diffusion of local anesthetics and vertebral canal anesthesia [17]. Fascia iliaca compartment block (FICB) has been defined as an anterior approach of the lumbar plexus block method, which is easy to perform and exhibits high safety and optimal analgesic effects. It has become increasingly accepted and it is widely used in lower limb surgeries in orthopedics departments [18]. Previous studies have reported that the failure rate of FICB, which aimed to block the lateral femoral cutaneous nerve, was approximately $10-37 \%$ due to the individualized variation, distribution and branching of nerves inferior to the inguinal ligament. Despite this evidence, the selection of the area above the inguinal ligament and below the pelvic iliac fascia is considered a reliable approach. Therefore, an applicable method modified from FICB, termed as SIFI block, was developed to replace the conventional fascia iliaca block and femoral nerve block [19]. In their study, Zheng et al. [11] used the SIFI compartment block in order to demonstrate that the diffusion of 30 $\mathrm{ml}$ local anesthetics could fully block the femoral nerve and lateral femoral cutaneous nerve, as well as provide a $56 \%$ block of the obturator nerve. This would popularize the use of the anterior approach lumbar plexus block.

The results of the present study indicated that the maximal pain score and time of position placing were significantly lower in the $S$ group than those of the $L$ group. We speculated that this could be due to the following reasons: 1) The stimulation during position placing could increase the pain and require cooperation among multiple operators; 2 ) the procedures of position placing in patients using an external fixator were more complex and more time-consuming and thus increased the pain stimulation.

The findings of the present study indicated that the time for lumbar plexus block and the number of puncture attempts significantly differed between the two groups. The SIFI compartment block involves several features, including superficial location and rapid and clear ultrasound imaging compared with the conventional posterior approach lumbar plexus block [20]. The FICN was performed for the patients in the $S$ group. The superficial layer of fascia iliaca compartment was covered by fascia lata and fascia iliaca and the deep layer was the iliopsoas muscle, through which the femoral nerve and lateral femoral cutaneous nerve were allowed to travel. The high-frequency linear array probe has a high resolution for superficial tissues and the images are very clear. Therefore, the ultrasound-guided procedures could easily aid the injection of local anesthetics to target specific sites, resulting in lower puncture attempts $[21,22]$. Consequently, the time for SIFI compartment block in the S group was shorter, while the effectiveness was better than that of the inferior-inguinal block, which was in agreement with previous studies [23]. In contrast to these observations, the patients in the $L$ group received posterior approach lumbar plexus block. Due to the deep position and complex anatomical structures, the puncture was relatively difficult and therefore the procedure time was longer [24].

The findings of the present study indicated that the time for sacral plexus block was significantly higher in the $S$ group than that in the $L$ group. Due to the deep position of the sacral plexus, the block was generally performed under the guidance of the ultrasound and a nerve stimulator. The anatomical position of the sacral plexus was deeper in the supine position and the puncture was more difficult, 
requiring a longer time for the procedure compared with that of the conventional sacral plexus block in the lateral position, which was in agreement with previous studies [12]. Generally, such procedures need to be performed by experienced and skilled anesthetists in order to identify the bone landmarks, such as anterior superior spine. Increased pressure is generally required during the procedures to reduce the thickness of subcutaneous adipose tissues, which makes the imaging of the deep nerves more sufficient. In addition, the bed was adjusted to uplift the affected body area of the patient for $15^{\circ}$, which increased the operation field, facilitated the procedures and saved time.

The findings of the present study indicated that the intraoperative hemodynamics, dose of sufentanil used in surgery, VAS score at different time points following surgery, the degree of satisfaction of the patients and the adverse events of the nerve block did not significantly differ between the two groups. Intubation via laryngeal mask was performed 30 min following nerve block for patients in both groups, which shortened the onset time of the nerve block effects. The onset of the nerve block effect could provide sufficient analgesic effects, thus meeting the surgery requirements and aiding patients to tolerate surgical stimulations, while the effects on hemodynamics were not substantial and the dose of sufentanil was reduced. In their study, Badiola et al. [25] suggested that the PACU pain scores in patients with moderate to severe pain following hip arthroscopic surgeries were not inferior in patients who received iliac fascia block than lumbar plexus block, which suggested that the analgesic effect of the iliac fascia block was similar to that of the lumbar plexus block. The combination with sacral plexus block could further improve the postoperative analgesic effects. Therefore, the VAS scores at different time points following surgery and the postoperative satisfactory degree to analgesia were similar between the two groups [26].

The present study contains certain limitations. The data were derived from two hospitals, which may limit the generalisability of our results. The sample size of the present study was small and a double-blind design was not used. The follow-up investigator may have been biased. The blockers were two anesthetists and may have performed operational errors.

\section{Conclusions}

In the present study, LSPB combined with venous anesthesia was used for patients in both groups and in different positions. This method led to anesthetic effects that allowed the completion of surgery and postoperative analgesia. The findings demonstrate that ultrasound-guided LSPB in the supine position is safe and applicable for lower limb surgeries. In addition, the advantage of LSPB in the supine position is the wider application scope with consistent anesthetic effects, higher comfort and better acceptance from patients, as well as the higher intraoperative satisfactory degree. These procedures can also safeguard the patients in the orthopedics departments from inconvenience and uncertainty due to repeated position changing. They may also avoid the fracture-dislocation and injuries of blood vessels and nerves induced by position placing, while the effects on hemodynamics are limited. Therefore, the procedures are simple to perform, with low side effects [27] and are considered applicable in clinical practice and worth of wide application. 


\section{Declarations}

\section{Ethics approval and consent to participate}

This randomized, blinded study was performed in compliance with the declaration of Helsinki and its amendments and was conducted according to the principles of Good Clinical Practice. The trial was registered prior to patient enrollment at the Chinese Clinical Trail Registry (11/03/2021

ChiCTR2100044117).The present study was approved by the Ethics Committee of the Chaohu Hospital Affiliated to Anhui Medical University (01/12/2020 202001-KYXM-01). All patients gave their written informed consent before participation.

\section{Consent for publication}

Not Applicable

\section{Availability of data and materials}

The datasets used and/or analysed during the current study are available from the corresponding author and editorial office on reasonable request.

\section{Competing interests}

The authors declare that they have no competing interests.

\section{Funding}

None

\section{Authors' contributions}

YX: Contributed substantially to study conception and design, execution of study, data acquisition, analysis, data interpretation first drafting. JS: Contributed substantially execution of study, data acquisition, interpretation of results. YL: Contributed substantially execution of study, data acquisition. YY: Contributed substantially execution of study, data acquisition. XX: Contributed substantially to study conception and design, and critical revision of the manuscript. XH: Contributed substantially to study conception and design, and critical revision of the manuscript. LW: Contributed substantially execution of study, interpretation of results. ZT: Contributed substantially to interpretation of data and critical revision of the manuscript. All authors read and approved the final manuscript.

\section{Acknowledgements}

First of all,I would like to extend my sincere gratitude to all the patients.I am also deeply indebted to all the other tutors and teachers in Translation Studies for their direct and indirect help to me.Finally,l am indebted to my parents for their continuous support and encouragement. 


\section{References}

1. Holloway KL, Yousif D, Bucki-Smith G,et al. Lower limb fracture presentations at a regional hospital. Arch Osteoporos 2017; 12(1):75.

2. Kaye JA, Jick H. Epidemiology of lower limb fractures in general practice in the United Kingdom. Inj Prev 2004; 10(6):368-74.

3. Ojo F, Al Snih S, Ray LA, Raji MA, Markides KS. History of fractures as predictor of subsequent hip and nonhip fractures among older Mexican Americans. J Natl Med Assoc 2007; 99(4):412-8.

4. Adler RA. Osteoporosis in men: a review. Bone Res 2014; 2:14001.

5. Pasco JA, Brennan SL, Henry MJ,et al. Changes in hip fracture rates in southeastern Australia spanning the period 1994-2007. J Bone Miner Res 2011; 26(7):1648-54.

6. Williams LJ, Pasco JA, Stuart AL,et al. Psychiatric disorders, psychotropic medication use and falls among women: an observational study. BMC Psychiatry 2015; $15: 75$.

7. Singh A, Lim ASM, Lau BPH, O'Neill G. Epidemiology of pelvic and acetabular fractures in a tertiary hospital in Singapore. Singapore Med J 2021.

8. Yang R, Liu RH, Xu JN,et al. Effects of Different Local Analgesic Techniques on Postoperative Quality of Life and Pain in Patients Undergoing Total Hip Arthroplasty Under General Anesthesia: A Randomized Controlled Trial. J Pain Res 2021; 14:527-36.

9. de Visme V, Picart F, Le Jouan R,et al. Combined lumbar and sacral plexus block compared with plain bupivacaine spinal anesthesia for hip fractures in the elderly. Reg Anesth Pain Med 2000; 25(2):15862.

10. Kirchmair L, Entner T, Kapral S, Mitterschiffthaler G. Ultrasound guidance for the psoas compartment block: an imaging study. Anesth Analg 2002; 94(3):706-10; table of contents.

11. Zheng T, Hu B, Zheng CY,et al. Improvement of analgesic efficacy for total hip arthroplasty by a modified ultrasound-guided supra-inguinal fascia iliaca compartment block. BMC Anesthesiol 2021; 21(1):75.

12. Wang $A Z$, Fan $\mathrm{K}$, Zhou $\mathrm{QH}$,et al. A lateral approach to ultrasound-guided sacral plexus block in the supine position. Anaesthesia 2018; 73(8):1043-4.

13. Karmakar MK, Li JW, Kwok WH, Soh E, Hadzic A. Sonoanatomy relevant for lumbar plexus block in volunteers correlated with cross-sectional anatomic and magnetic resonance images. Reg Anesth Pain Med 2013; 38(5):391-7.

14. Bendtsen TF, Lönnqvist $P A$, Jepsen $\mathrm{KV}$,et al. Preliminary results of a new ultrasound-guided approach to block the sacral plexus: the parasacral parallel shift. Br J Anaesth 2011; 107(2):278-80.

15. Scurrah A, Shiner CT, Stevens JA, Faux SG. Regional nerve blockade for early analgesic management of elderly patients with hip fracture - a narrative review. Anaesthesia 2018; 73(6):769-83.

16. Liu Y, Ke X, Wu X, Mei W. Ultrasound-guided Lumbar Plexus Block in Supine Position. Anesthesiology 2018; 128(4):812. 
17. Saranteas T, Anagnostopoulos D, Kostroglou A, Souvatzoglou R. The "Shamrock method" for ultrasound-guided lumbar plexus nerve block in the supine position. J Clin Anesth 2021; 71:110249.

18. Haslam L, Lansdown A, Lee J, van der Vyver M. Survey of Current Practices: Peripheral Nerve Block Utilization by ED Physicians for Treatment of Pain in the Hip Fracture Patient Population. Can Geriatr J 2013; 16(1):16-21.

19. Zhao J, Huang Y, Fu M, Tao F. Ultrasound-guided suprainguinal fascia iliaca block combined with a sacral plexus block for lower extremity surgery: A case report. Medicine (Baltimore) 2020; 99(35):e21921.

20. Bravo D, Layera S, Aliste J,et al. Lumbar plexus block versus suprainguinal fascia iliaca block for total hip arthroplasty: A single-blinded, randomized trial. J Clin Anesth 2020; 66:109907.

21. Højer Karlsen AP, Geisler A, Petersen PL, Mathiesen O, Dahl JB. Postoperative pain treatment after total hip arthroplasty: a systematic review. Pain 2015; 156(1):8-30.

22. Dolan J, Williams A, Murney E, Smith M, Kenny GN. Ultrasound guided fascia iliaca block: a comparison with the loss of resistance technique. Reg Anesth Pain Med 2008; 33(6):526-31.

23. Vermeylen K, Desmet M, Leunen I,et al. Supra-inguinal injection for fascia iliaca compartment block results in more consistent spread towards the lumbar plexus than an infra-inguinal injection: a volunteer study. Reg Anesth Pain Med 2019.

24. Min L, Chen C, Yan Y,et al. Comparison of Combined Lumbosacral Plexus and T12 Paravertebral Nerve Blocks With General Anesthesia in Older Adults Undergoing Primary Total Hip Arthroplasty: A Retrospective, Propensity Score-Matched Cohort Study. Geriatr Orthop Surg Rehabil 2020; 11:2151459320976531.

25. Badiola I, Liu J, Huang S, Kelly JDt, Elkassabany N. A comparison of the fascia iliaca block to the lumbar plexus block in providing analgesia following arthroscopic hip surgery: A randomized controlled clinical trial. J Clin Anesth 2018; 49:26-9.

26. Desmet $M$, Vermeylen $K$, Van Herreweghe I,et al. A Longitudinal Supra-Inguinal Fascia Iliaca Compartment Block Reduces Morphine Consumption After Total Hip Arthroplasty. Reg Anesth Pain Med 2017; 42(3):327-33.

27. Yang S, Chen K, Wan L. Combination of ultrasound-guided lumbosacral plexus block with anterior quadratus lumborum block in supine position for hip surgery: a case report. J Anesth 2020; 34(5):777-80.

\section{Figures}

\section{Figure 1}

CONSORT diagram of patient flow through the study. 


\section{Figure 2}

(A) A patient in the $S$ group who was in the supine position for iliac plexus block; (B) ultrasound image of the patient (A) who underwent iliac plexus block. (C) A patient in the S group who was in the supine position for sacral plexus block; (D) ultrasound image of the patient (C) who underwent sacral plexus block. (E) A patient in the $L$ group in the lateral position for LSPB; $(F)$ ultrasound image of the patient (E) who underwent iliac plexus block. $(G)$ Ultrasound image of the patient in the $L$ group who underwent sacral plexus block. ASIS: anterior superior iliac spine; IOM: internal oblique muscle; SM: sartorius muscle; IM: iliacus muscle; ESM: erector spinae muscle; TP: transverse process; ultrasound probe.

\section{Supplementary Files}

This is a list of supplementary files associated with this preprint. Click to download.

- Trialplan.docx 